\title{
Partial Deficiency of Emerin Caused by a Splice Site Mutation in EMD
}

\author{
Junhui Yuan ${ }^{1}$, Masahiro Ando ${ }^{1}$, Itsuro Higuchi ${ }^{1}$, Yusuke Sakiyama ${ }^{1}$, Eiji Matsuura ${ }^{1}$, \\ Kumiko Michizono ${ }^{1}$, Osamu Watanabe ${ }^{1}$, Shinjiro Nagano ${ }^{2}$, Yukie Inamori ${ }^{1}$, \\ Akihiro Hashiguchi ${ }^{1}$, Yujiro Higuchi ${ }^{1}$, Akiko Yoshimura ${ }^{1}$ and Hiroshi Takashima ${ }^{1}$
}

\begin{abstract}
Emery-Dreifuss muscular dystrophy (EDMD) is caused by mutations in the EMD gene on the $\mathrm{X}$ chromosome, which codes for emerin, an inner nuclear membrane protein. Monoclonal antibodies against the $\mathrm{N}$ terminus of emerin protein are used to screen for emerin deficiency in clinical practice. However, these tests may not accurately reflect the disease in some cases. We herein describe the identification of a splice site mutation in the EMD gene in a Japanese patient who suffered from complete atrioventricular conduction block, mild muscle weakness and joint contracture, and a persistently elevated serum creatine kinase level. We used multiple antibodies to confirm the presence of a novel truncating mutation in emerin without the transmembrane region and $\mathrm{C}$-terminus in the skeletal muscle.
\end{abstract}

Key words: Emery-Dreifuss muscular dystrophy (EDMD), splice site mutation, emerin, immunohistochemical stain

(Intern Med 53: 1563-1568, 2014)

(DOI: 10.2169/internalmedicine.53.8922)

\section{Introduction}

Emery-Dreifuss muscular dystrophy (EDMD) is characterized by early contractures of the elbow, neck, and Achilles tendons; slowly progressive skeletal muscle wasting in the upper arms and lower legs (humeroperoneal); and cardiomyopathy associated with conduction defects (1). The most serious aspect of EDMD is cardiac involvement, which usually becomes evident as muscle weakness progresses, but it may occur before there is any significant skeletal muscle involvement. EDMD is caused by mutations in different genes; including those in the EMD gene, encoding emerin, and causing X-linked EDMD (2); mutations in the LMNA gene, which encodes lamins $\mathrm{A}$ and $\mathrm{C}$, and causes autosomal dominant EDMD (3) and a very rare autosomal recessive EDMD (4).

Monoclonal antibodies against the N-terminus of emerin, including Novocastra ${ }^{\mathrm{TM}}$ Lyophilized Mouse Monoclonal An- tibody Emerin (NCL-emerin), have been used to screen emerin deficiency in clinical practice. In general, previous studies only used one kind of antibody in western blot or immunocytochemistry tests to evaluate emerin expression. Most of the mutations (86\%) affecting males result in a complete absence of emerin (5); however, the corresponding genotypes were different, indicating that pathological assessments using a single antibody could not accurately reflect patients' diverse genetic information.

We herein describe the case of a patient with typical complete atrioventricular conduction block along with mild muscle weakness and joint contracture. We identified a previously reported splice acceptor site mutation in $E M D$, but the original symptoms and clinical severity of our case are different from those of the previous patient with this mutation (6). Furthermore, immunohistochemistry using three antibodies against different emerin domains revealed a distinct partial deficiency of emerin in the nuclei of the skeletal muscle fibers.

\footnotetext{
${ }^{1}$ Department of Neurology and Geriatrics, Kagoshima University Graduate School of Medical and Dental Sciences, Japan and ${ }^{2}$ Department of Cardiology, Imamura Bun-in Hospital, Japan

Received for publication September 5, 2012; Accepted for publication January 30, 2014

Correspondence to Dr. Hiroshi Takashima, thiroshi@m3.kufm.kagoshima-u.ac.jp
} 


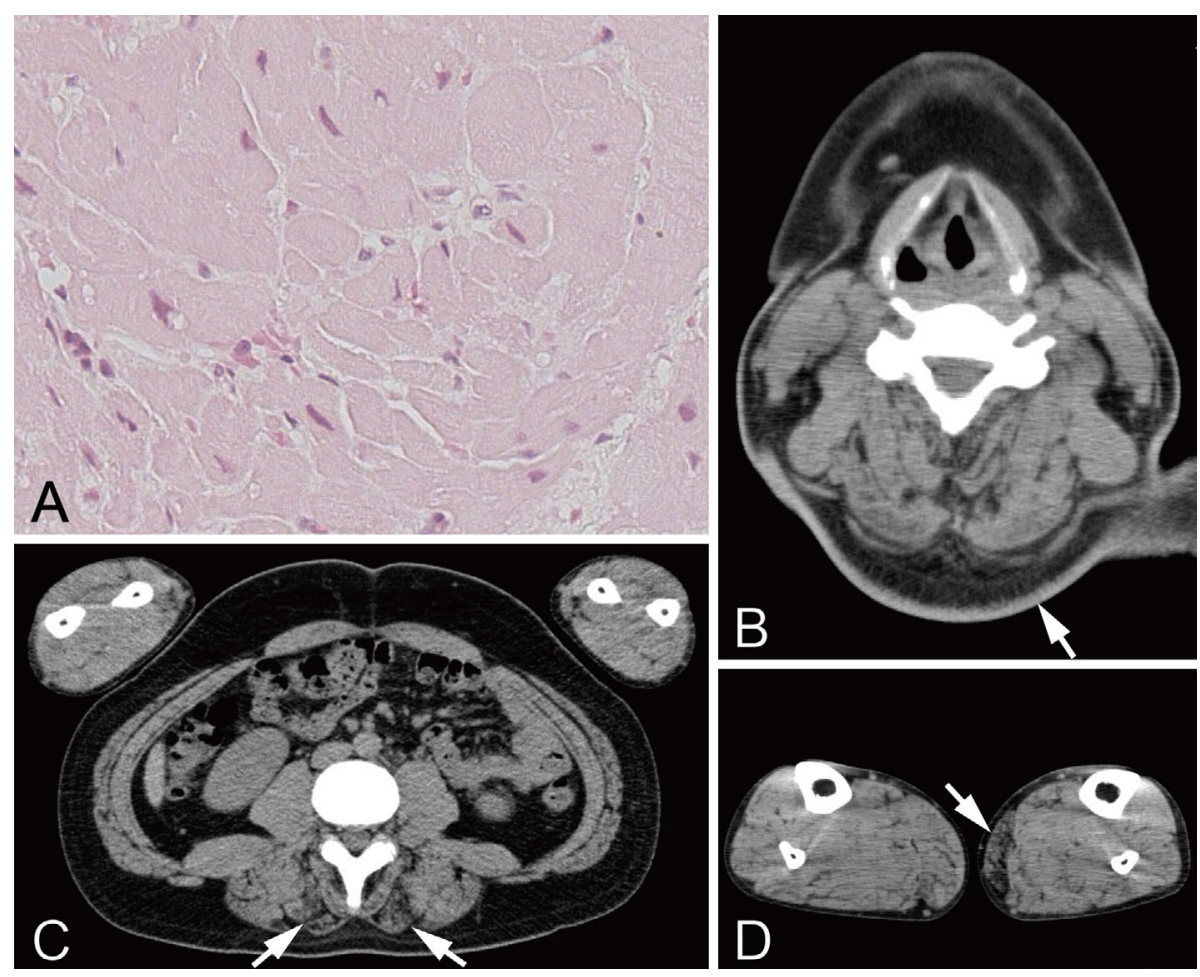

Figure 1. Cardiac pathology and CT findings of the paraspinal muscles and gastrocnemius. Hematoxylin and Eosin staining revealed mild myocardial interstitial fibrosis $(\times 800)($ A). A CT revealed low-density areas and atrophic changes (arrows) in the cervical and lumbar paraspinal muscles (B, C) and the left gastrocnemius (D).

\section{Case Report}

A 22-year-old male with normal growth and development was referred to our hospital with a complaint of chest discomfort at the age of 19 years. He had a complete atrioventricular conduction block (heart rate, 38/min), and his serum creatine kinase $(\mathrm{CK})$ was elevated to $3,000 \mathrm{IU} / \mathrm{mL}$ (normal range $45-163 \mathrm{IU} / \mathrm{mL}$ ). A chest X-ray and echocardiography (ECG) revealed no cardiomegaly. His symptoms gradually worsened, and symptoms of dizziness appeared due to bradycardia. The R-R interval of the ECG was extended to 9.2 s. Six months after the initial diagnosis, the patient was implanted with a permanent pacemaker. A sample of the left ventricular myocardium was obtained during cardiac catheterization, and histochemical staining revealed mild myocardial interstitial fibrosis without fiber disarray, fibril deposition, or myocardial injury (Fig. 1A). No abnormalities were detected in the respiratory function tests. Although he had suffered from diplopia and strabismus several years earlier, a neurological examination on admission showed normal ocular movement. His cognition, coordination, and facial muscle strength were also normal. The patient's neck flexion was slightly weak and was limited due to mild neck contracture. There was no scoliosis or contracture of any other joints in the extremities. His extremities showed no significant muscle weakness, atrophy, or sensory disturbances. Serum CK fluctuated between $1,600 \mathrm{IU} / \mathrm{mL}$ and $3,000 \mathrm{IU} / \mathrm{mL}$.
Other routine serologic evaluations and tests for serum autoantibodies revealed no abnormalities. Using electromyography, short-duration and some unstable polyphasic motor unit potentials were detected in the right biceps brachii, but no abnormality was found in the muscles of the lower extremities. A whole-body computed tomography (CT) scan revealed low-density areas and atrophic changes in the cervical and lumbar paraspinal muscles and the left gastrocnemius (Fig. 1B, C, D).

His 48-year-old mother suffered from a first-degree atrioventricular conduction block along with paroxysmal atrial fibrillation, and was treated with warfarin. In addition, unspecified abnormal ECGs were observed when his 24year-old twin elder sisters were screened, but no abnormalities could be detected in the workup. Periodic health examinations were advised for all three of his family members. Muscle CT examination was not performed in these probable female carriers.

The study protocol was reviewed and approved by the Institutional Review Board of Kagoshima University. The patient provided his written, informed consent to participate in this study.

\section{Pathological studies}

A skeletal muscle sample was obtained from the biceps brachii, but no skin sample was collected. Histochemical staining revealed mild variations in fiber diameter, a few degenerating or regenerating fibers, a slight increase in the 


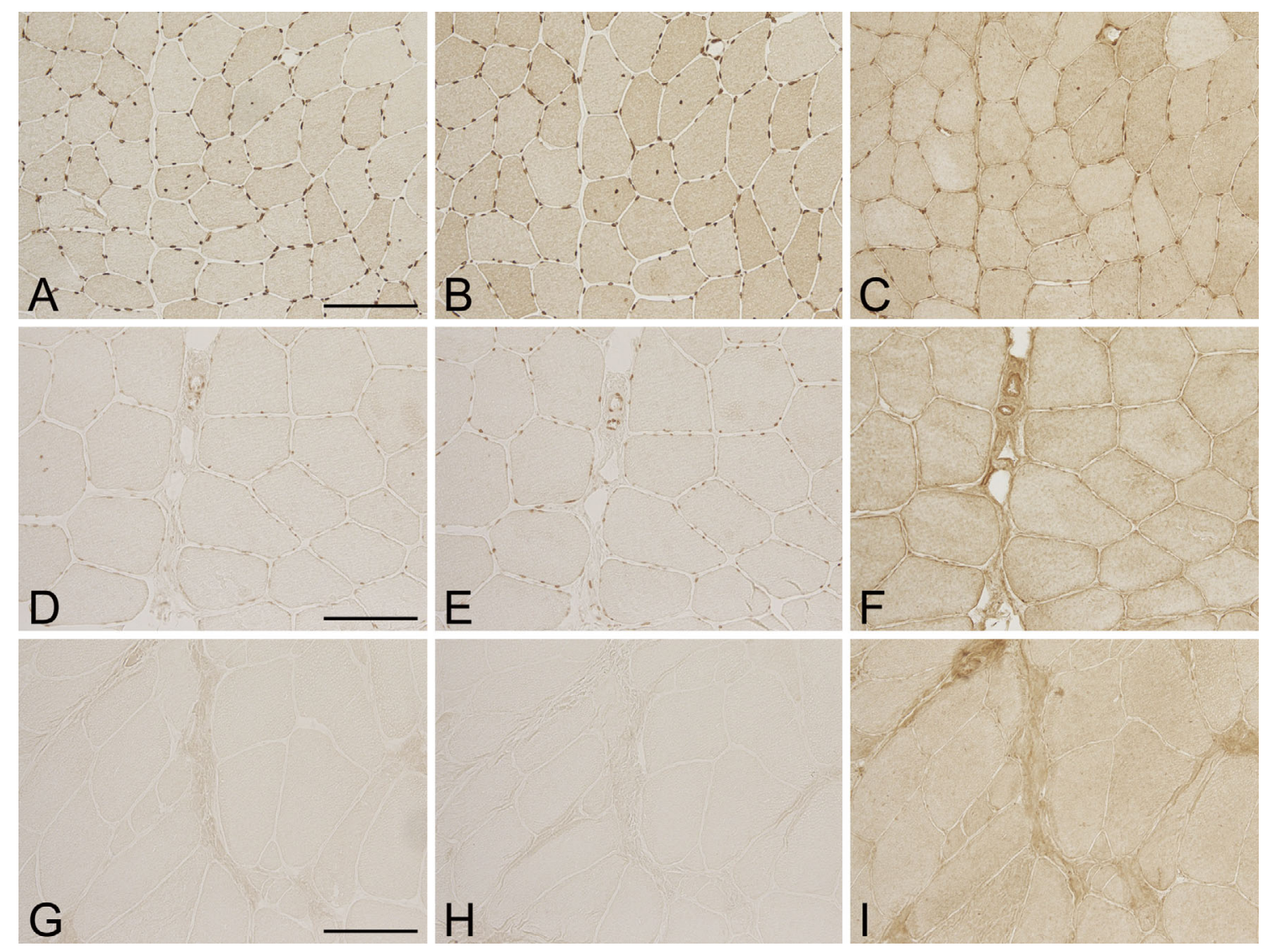

Figure 2. Serial immunohistochemical staining with NCL-Emerin (A, D, G), H-12 (B, E, H), and C-20 (C, F, I) antibodies. A-C: Emerin was strongly expressed on the nuclei of skeletal muscle fibers in the healthy control subject. D-F: In the patient, emerin expression was significantly decreased as detected by NCL-Emerin and H-12 labeling, and it was completely absent as assessed by C-20 labeling. G-I: In the disease control, staining experiments revealed a complete absence of emerin with all three antibodies. No counterstain was used. Bar $=100 \mu \mathrm{m}$

number of internal nuclei, and slight connective tissue proliferation. The number of hypertrophic fibers was increased. Immunohistochemical stains were performed manually with a 1:50 dilution of NCL-Emerin (Novocastra, Leica Microsystems, Newcastle Upon Tyne, UK), H-12 (Santa Cruz Biotechnology, Santa Cruz, USA) monoclonal antibodies for the $\mathrm{N}$-terminus of emerin, and a 1:20 dilution of C-20 affinity purified polyclonal antibody for the C-terminus of emerin (Santa Cruz Biotechnology). A skeletal muscle specimen from an unrelated 39-year-old male with typical Xlinked EDMD was used as a disease control. The three antibodies detected emerin expression on the nuclei of the skeletal muscle in the healthy control subject. In our patient, emerin expression was significantly decreased as detected by NCL-Emerin and H-12 labeling, and it was completely absent with C-20 labeling. In the disease control specimen, nuclear staining was absent with all three antibodies against emerin (Fig. 2). In western blot analyses using the three antibodies, we could not detect any clear bands in the patient's samples, and this was probably due to the low expression of protein or detection sensitivity of western blot (data not shown).

\section{Genetic studies}

Genomic DNA was extracted from the patient's blood lymphocytes. We were not able to obtain consent for genetic analysis from his three family members or the disease control patient. Using the Primer3 (v. 0.4.0) online program (http://frodo.wi.mit.edu/), we designed oligonucleotide primers flanking the six exons and intron-exon junctions in the $E M D$ gene. After hot-start polymerase chain reaction (PCR) amplification, the products were sequenced by dyeterminator chemistry using an ABI3010 sequencer (Applied Biosystems, Foster City, USA). A splice acceptor site mutation located at the second nucleotide before exon six (c.450$2 \mathrm{~A}>\mathrm{G})$ of $E M D$ was identified. This created a new restriction site for AvaI. Using the forward (5'-CTCGCCCTGACT CTCTTCTG-3') and reverse (5'-CTAAGGCAGTCAGCCAG GAC-3') primers, a 533-bp PCR product covering this mutation was amplified and was divided into $385 \mathrm{bp}$ and $148 \mathrm{bp}$ fragments (Fig. 3A, B). This mutation was not detected in 100 Japanese control chromosomes, and we did not find the c. $450-2 \mathrm{~A}>\mathrm{G}$ mutation in the 1,000 Genomes database that catalogs human genetic variations in 2,500 samples, including 500 East Asian (100 Japanese) samples (http:// 


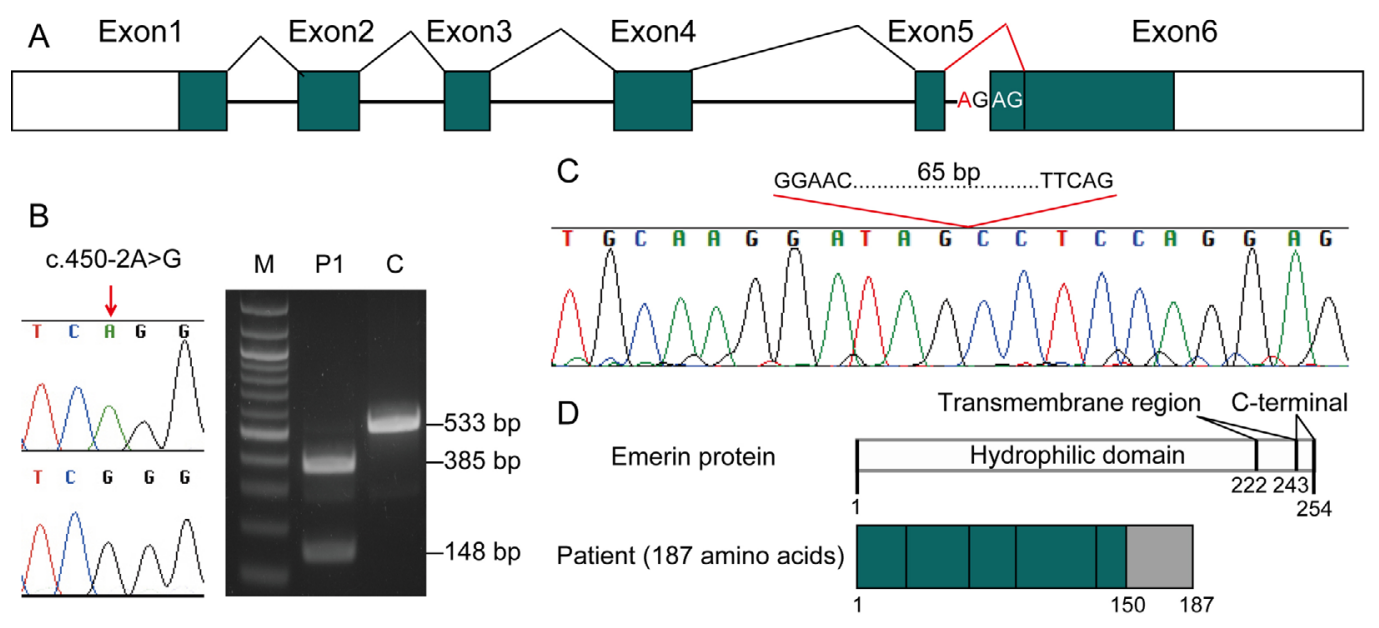

Figure 3. Genetic studies and an ideogram of the aberrant splicing. A: The splice acceptor site mutation located at the second nucleotide before exon 6 (red: c.450-2A) of the EMD gene. B: Sequencing chromatogram of the c.450-2A > G mutation in EMD (red arrow) and the amplicon (533 bp) covering exon 6, which was divided into 385-bp and 148-bp fragments by AvaI. C: This aberrant splicing eliminated 65 nucleotides in the transcript of exon 6. D: The mutation shifted the reading frame (gray column) and produced a truncated transcript of 187 amino acids without the transmembrane region and C-terminal tail.

brower.1000genomes.org).

Total RNA was isolated and purified from the biopsied skeletal muscle specimen using a RecoverAll ${ }^{\mathrm{TM}}$ Total Nucleic Acid Isolation Kit (Ambion, Austin, USA). Complementary DNA (cDNA) was generated by reverse transcription PCR (RT-PCR) of the isolated RNA $(1 \mu \mathrm{g})$ using a High Capacity cDNA Reverse Transcription Kit (Applied Biosystems) according to the manufacturer's instructions. The cDNA was subsequently amplified with the forward (5'-ACCAGAGCAAGGGCTACAATGACG-3') and reverse primers (5'-GGTGGCCTTTGGTTAATCCCCTC-3') using KOD FX (Toyobo, Osaka, Japan). No band was detected at the expected position of the full-length emerin transcript, and a unique shorter band was extracted from the gel (QIAquick Gel Extraction Kit, Qiagen, Venlo, Netherlands) and sequenced. The first 65 nucleotides in exon six were found to be absent, and the reading frame was shifted. The protein was changed because the frame was shifted from position 150 to a premature stop codon at 187 (p.Arg150fs), producing a truncated emerin protein lacking the transmembrane region and C-terminal tail (Fig. 3C, D).

\section{Discussion}

We studied the clinical, pathological, and genetic features of a Japanese patient with X-linked EDMD. We observed a partial deficiency of emerin in the skeletal muscle with severe arrhythmia and continuous serum CK elevation, but he only exhibited slight muscular involvement and mild joint contracture. A splice acceptor site mutation, c.450-2A $>\mathrm{G}$ (p.Arg150fs), was identified in the $E M D$ gene of this patient.

The EMD gene on chromosome $\mathrm{Xq} 28$ contains six exons and encodes an emerin protein with 254 amino acids. Emerin consists of a large hydrophilic nucleoplasmic domain (residues 1-222), a transmembrane region (residues 223-243), and a short C-terminal tail (residues 244-254) (7). It is a ubiquitously expressed protein located at the inner nuclear membrane of most cells in the body with the highest mRNA expression in skeletal and cardiac muscle, and it has been proposed to have functions in gene expression, RNA processing, cell signaling, and chromatin dynamics $(2,8)$.

The 22-year-old patient in this study suffered a complete atrioventricular conduction block, and a permanent pacemaker was implanted to prevent lethal cardiac arrest. His mother and sisters were also affected with arrhythmia, but they did not show evidence of skeletal muscle abnormalities. Previous reports have documented that such symptomatic female carriers have very low emerin levels $(<5 \%$ of normal) due to skewed X-inactivation (9). A pathological study of cardiac muscle performed by local doctors revealed nonspecific mild interstitial fibrosis. Slight muscular involvement was observed during both the clinical and imaging examinations. The cause of this proband was unclear until his persistent high CK level was noticed and cardioskeletal myopathy suspected, at which point a skeletal muscle specimen was obtained.

The histochemical pathology of the skeletal muscle indicated fiber size variation with scattered internalized nuclei. An immunohistochemical study with the NCL-Emerin monoclonal antibody targeted to 220 amino acids near the Nterminus of emerin revealed a significant reduction of nuclear emerin, and a diagnosis of X-linked EDMD was made. After direct sequencing of the $E M D$ gene, an $\mathrm{A}>\mathrm{G}$ transversion at 1,644 (c.450-2A>G) was observed, which should eliminate the splice acceptor site before exon six. In order to 
identify the candidate acceptor site, we performed RT-PCR using mRNA extracted from the patient's frozen skeletal muscle tissue. A truncated transcript with 187 residues generated by aberrant splicing was detected. The new AG splice acceptor site, which occurs 65 bp after the wild-type site in the $E M D$, shifted the frame from position 150 to a premature stop codon at position 187. Most of the truncated emerin lacked the transmembrane segment and the Cterminal tail, thus suggesting that it might fail to target the endoplasmic reticulum and would thus be rapidly degraded (10). Interestingly, this c. $450-2 \mathrm{~A}>\mathrm{G}$ splice site mutation was reported in another unrelated patient in Japan in 1999 (6). The initial symptom of the first patient was neck contracture, but our patient initially experienced cardiac involvement. In fact, immunostaining and immunoblotting studies performed in the previous patient revealed the absence of emerin and a premature stop codon generated at residue 235 . The differences between these two patients with same mutation suggest that there could be other potential mechanisms involved in protein expression and mRNA splicing.

In an additional immunohistochemical study, we employed two additional emerin-targeted antibodies, H-12 (targets residues 3 to 254) and C-20 (targets a peptide near the C-terminus). In the present patient, emerin expression was detected by H-12 staining (but at a much lower level than normal) but not by C-20 staining. In this case, a skin sample could have been helpful in further verifying the status of emerin. We concluded that the truncated emerin in the present patient could react with antibodies targeting a peptide near the N-terminus (NCL-Emerin and $\mathrm{H}-12$ ) at much lower levels, but it could not bind the antibody targeted to the Cterminus (C-20). In addition, the nucleoplasm showed stronger staining than the cytoplasm, but this was not as strong as that observed at the nuclear edge, thus indicating that emerin may have been expressed in the nucleoplasm. An immunoelectron microscopy study might be more reliable for identifying emerin expression. The reason why the mutated version of emerin without the transmembrane segment was detectable on the nuclei requires further research.

According to the UMD-EMD mutations database (http:// www.umd.be/EMD/), 94 different mutations of the EMD gene have been reported in 298 records, and 15 of these are splice site mutations (15.96\%). It is notable that some other $\mathrm{X}$-linked EDMD patients with truncated emerin longer than 187 amino acids presented with complete absences of emerin as detected with the NCL-Emerin antibody from the same company (11-13). To evaluate the emerin protein expression, it would be more reliable to use various antibodies targeted to different domains. An antibody targeting the Cterminus could therefore be useful for detecting truncated emerin, which accounts for most EMD mutations.

Mutations in emerin can cause different phenotypes, even within the same family. In patients with distal premature stop codons, which do not evoke a nonsense-mediated decay mechanism, the phenotypes present as a typical triad $(6,12-16)$ or cardiac phenotype $(11,17)$. Rare cases of a reduction in emerin due to a missense mutation may have a milder phenotype (18). The cardiac muscle is supposed to be less tolerant of emerin deficiency than the skeletal muscle (19). A lack of or decrease in emerin levels in the heart may alter electrical resistance and cardiomyocyte adhesion, which could lead to conduction delay or block (17). Emerinopathy also includes a limb-girdle muscular dystrophy phenotype $(20,21)$. In the present case with partial emerin deficiency, although a complete atrioventricular conduction block was observed, there was only slight muscle weakness and joint contracture. The truncated protein remaining in the nucleoplasm may have contributed to the relatively benign phenotype, especially in the skeletal muscle.

In conclusion, we identified a splice site mutation before the last exon of EMD in an X-linked EDMD patient, and he showed evidence of a truncated protein at both pathological and genetic levels. We detected partial expression of a truncated emerin protein without the transmembrane region and C-terminal domain by immunohistochemical staining with antibodies against different emerin domains. We recommend the use of multiple antibodies that bind different emerin domains in order to obtain a comprehensive view of the protein. The anti-emerin antibody C-20 may be useful for detecting the absence of the C-terminus. Our findings also suggest that all young patients with significant cardiac conduction defects of unknown etiology be assessed to exclude the presence of EDMD, even if they have either no skeletal muscle or only slight skeletal muscle or joint involvement.

\section{Author's disclosure of potential Conflicts of Interest (COI).}

Hiroshi Takashima: Patent royalties/licensing fees, Achena Diagnosis

\section{Acknowledgement}

The authors thank Ms. N. Hirata of our department for her excellent technical assistance. We also wish to thank the Joint Research Laboratory, Kagoshima University Graduate School of Medical and Dental Sciences for the use of their facilities.

Financial Support: This project was funded by the Ministry of Education, Culture, Sports, Science and Technology of Japan (grant 21591094, IH); Intramural Research Grant (23-5) for Neurological and Psychiatric Disorders of NCNP Applying Health and Technology, from the Ministry of Health, Labour and Welfare of Japan; and a research grant (23300201) from the Ministry of Health, Labour and Welfare of Japan.

\section{References}

1. Emery AE, Dreifuss FE. Unusual type of benign $x$-linked muscular dystrophy. J Neurol Neurosurg Psychiatry 29: 338-342, 1966.

2. Bione $S$, Maestrini $E$, Rivella $S$, et al. Identification of a novel Xlinked gene responsible for Emery-Dreifuss muscular dystrophy. Nat Genet 8: 323-327, 1994.

3. Bonne G, Di Barletta MR, Varnous S, et al. Mutations in the gene 
encoding lamin A/C cause autosomal dominant Emery-Dreifuss muscular dystrophy. Nat Genet 21: 285-288, 1999.

4. Raffaele Di Barletta M, Ricci E, Galluzzi G, et al. Different mutations in the LMNA gene cause autosomal dominant and autosomal recessive Emery-Dreifuss muscular dystrophy. Am J Hum Genet 66: $1407-1412,2000$

5. Helbling-Leclerc A, Bonne G, Schwartz K. Emery-Dreifuss muscular dystrophy. Eur J Hum Genet 10: 157-161, 2002.

6. Funakoshi M, Tsuchiya Y, Arahata K. Emerin and cardiomyopathy in Emery-Dreifuss muscular dystrophy. Neuromuscul Disord 9: 108-114, 1999.

7. Ellis JA, Brown CA, Tilley LD, et al. Two distal mutations in the gene encoding emerin have profoundly different effects on emerin protein expression. Neuromuscul Disord 10: 24-30, 2000.

8. Holaska JM, Wilson KL. An emerin "proteome": purification of distinct emerin-containing complexes from HeLa cells suggests molecular basis for diverse roles including gene regulation, mRNA splicing, signaling, mechanosensing, and nuclear architecture. Biochemistry 46: 8897-8908, 2007.

9. Manilal S, Recan D, Sewry CA, et al. Mutations in EmeryDreifuss muscular dystrophy and their effects on emerin protein expression. Hum Mol Genet 7: 855-864, 1998.

10. Yates JR, Bagshaw J, Aksmanovic VM, et al. Genotype-phenotype analysis in X-linked Emery-Dreifuss muscular dystrophy and identification of a missense mutation associated with a milder phenotype. Neuromuscul Disord 9: 159-165, 1999.

11. Vytopil M, Vohanka S, Vlasinova J, et al. The screening for $X-$ linked Emery-Dreifuss muscular dystrophy amongst young patients with idiopathic heart conduction system disease treated by a pacemaker implant. Eur J Neurol 11: 531-534, 2004.

12. Astejada MN, Goto $K$, Nagano A, et al. Emerinopathy and laminopathy clinical, pathological and molecular features of muscular dystrophy with nuclear envelopathy in Japan. Acta Myol 26:
159-164, 2007.

13. Paradas C, Márquez C, Gallardo E, et al. X-linked Emery-Dreifuss muscular dystrophy and vacuoles: an immunohistochemical characterization. Muscle Nerve 32: 61-65, 2005.

14. Ognibene A, Sabatelli P, Petrini $S$, et al. Nuclear changes in a case of X-linked Emery-Dreifuss muscular dystrophy. Muscle Nerve 22: 864-869, 1999.

15. Hong JS, Ki CS, Kim JW, et al. Cardiac dysrhythmias,cardiomyopathy and muscular dystrophy in patients with EmeryDreifuss muscular dystrophy and limb-girdle muscular dystrophy type 1B. J Korean Med Sci 20: 283-290, 2005.

16. Niebroj-Dobosz I, Marchel M, Madej A, et al. Circulating autoantibodies to troponin I in Emery-Dreifuss muscular dystrophy. Acta Myol 27: 1-6, 2008.

17. Sakata K, Shimizu M, Ino H, et al. High incidence of sudden cardiac death with conduction disturbances and atrial cardiomyopathy caused by a nonsense mutation in the STA gene. Circulation 111: 3352-3358, 2005.

18. Ellis JA, Yates JR, Kendrick-Jones J, Brown CA. Changes at P183 of emerin weaken its protein-protein interactions resulting in $\mathrm{X}$ linked Emery-Dreifuss muscular dystrophy. Hum Genet 104: 262268, 1999.

19. Wehnert M, Muntoni F. 60th ENMC international workshop: non X-linked Emery-Dreifuss muscular dystrophy 5-7 June 1998, Naarden, The Netherlands. Neuromuscul Disord 9: 115-121, 1999.

20. Muntoni F, Lichtarowicz-Krynska EJ, Sewry CA, et al. Early presentation of X-linked Emery-Dreifuss muscular dystrophy resembling limb-girdle muscular dystrophy. Neuromuscul Disord 8: 7276, 1998.

21. Ura S, Hayashi YK, Goto K, et al. Limb-girdle muscular dystrophy due to emerin gene mutations. Arch Neurol 64: 1038-1041, 2007.

(C) 2014 The Japanese Society of Internal Medicine http://www.naika.or.jp/imonline/index.html 